\title{
Desnutrición hospitalaria en una institución privada de la Ciudad de México. Lo que hay después del tamizaje
}

\author{
Hospital malnutrition in a private institution in Mexico City. \\ What happens after nutritional screening \\ Desnutrição hospitalar em uma instituição particular na Cidade de México. \\ O que há apos a triagem
}

Adriana Vázquez Callejas ${ }^{1 *}$, Quetzalina Flores Fajardo', Jorge Chirino Romo²

Recibido: 27 de marzo de 2019. Aceptado para publicación: 5 de septiembre de 2019.

Publicado en línea: 9 de noviembre de 2019

https://doi.org/10.35454/rncm.v3n1.024

\section{Resumen}

Introducción: los métodos de tamizaje y diagnóstico nutricional para la detección de pacientes con riesgo de desnutrición o desnutrición son ampliamente utilizados al ingreso hospitalario. Sin embargo, su beneficio es limitado si no se acompaña de una intervención adecuada.

Objetivo: identificar el porcentaje de pacientes que recibieron tratamiento nutricional después del proceso de tamizaje al ingreso hospitalario en una institución privada de Ciudad de México.

Métodos: estudio observacional, retrospectivo, transversal y descriptivo. Se revisaron los expedientes de pacientes mayores de 18 años y estancia intrahospitalaria mayor a 7 días, y a quienes se les realizó Valoración Global Subjetiva en las primeras 48 horas del ingreso en el periodo de 01 de enero a 30 de junio del 2017.

Resultados: en una muestra de 198 expedientes, se encontró una prevalencia de riesgo de desnutrición y desnutrición moderada/severa de $83,8 \%$. De ellos, solo $8,4 \%$ recibió una evaluación nutricional integral, y a $28,9 \%$ se le ofreció terapia nutricional.

Conclusión: la prevalencia de desnutrición en un hospital privado en la Ciudad de México es alta. La mayoría de estos pacientes no reciben evaluación ni

\section{Summary}

Introduction: Screening tools are widely used for the detection of patients at risk of malnutrition or malnutrition at hospital admission. However, the usefulness of the nutritional screening is limited if it is not accompanied by an appropriate intervention.

Objective:To evaluate how many patients with malnutrition or risk of malnutrition were managed with nutritional treatment in a private hospital in Mexico City.

Methods: Observational, retrospective, cross-sectional and descriptive study. Review of records of patients hospitalized in a third level private hospital in Mexico City. Inclusion: $>18$ years, $\geq 7$ days of inpatient hospital stay, with a Subjective Global Assessment in the first 48 hours of admission.

Results: From a sample of 198 records, a prevalence of risk of malnutrition and moderate / severe malnutrition of $83.8 \%$ was found. Of these, only $8.4 \%$ received a comprehensive nutritional evaluation. The $28.9 \%$ received nutritional therapy.

Conclusion: There is a high prevalence of malnutrition in patients admitted to a private hospital in Mexico City. The majority of these patients do not receive evaluation or specialized nutritional treatment.

\section{Resumo}

Introdução: as ferramentas de triagem são amplamente utilizadas para a deteção de pacientes com risco de desnutrição na admissão hospitalar. No entanto, a utilidade de triagem nutricional é limitada se não for acompanhada de uma intervenção apropriada.

Objetivo: avaliar quantos pacientes com desnutrição ou risco de desnutrição receberam tratamento nutricional em um hospital privado na Cidade do México.

Métodos: estudo observacional, retrospetivo, transversal e descritivo. Revisão do registo de pacientes internados em um hospital privado de terceiro nível na Cidade de México. Inclusão: > 18 anos, $\geq 7$ dias de internamento hospitalar, com uma Avaliação Global Subjetiva nas primeiras 48 horas de ingresso.

Resultados: em una amostra de 198 registos, foi encontrada uma prevalência de risco de desnutrição e desnutrição moderada / grave de $83,8 \%$. Destes, apenas $8,4 \%$ receberam uma avaliação nutricional objetiva. 28,9\% receberam terapia nutricional.

Conclusão: existe alta prevalência de desnutrição nos pacientes internados num hospital privado na Cidade de México. A maioria desses pacientes não recebeu avaliação ou tratamento nutricional 
tratamiento nutricional especializado. Se requiere implementar mejores estrategias para la atención multidisciplinaria en los pacientes con desnutrición.

Palabras clave: desnutrición, tamizaje, terapia nutricional.
It is necessary to implement better strategies for multidisciplinary care in patients with malnutrition.

Keywords: Malnutrition; Screening; Nutrition therapy. especializado. É necessário implementar melhores estratégias para o entendimento multidisciplinar em pacientes com desnutrição.

Palavras-chave: desnutrição, triagem, terapia nutricional.
Nutriología Clínica Hospital Ángeles del Pedregal, Ciudad de México.

2 Cirugía General y Nutriología Clínica Hospital Ángeles del Pedregal, Ciudad de México
*Correspondencia: Adriana Vázquez Callejas adrivazquezc@hotmail.com

\section{INTRODUCCIÓN}

La desnutrición es frecuente en pacientes hospitalizados. Esta condición se relaciona con aumento del riesgo de complicaciones infecciosas y no infecciosas, aumento de días de estancia hospitalaria, costos, morbilidad y mortalidad ${ }^{(1-4)}$. Los factores que favorecen la aparición de desnutrición en el paciente hospitalizado incluyen, por un lado, los propios de la enfermedad, como un estado inflamatorio y catabólico, anorexia, alteraciones metabólicas, alteraciones de los sentidos, interacciones con medicamentos, etcétera. Y, por otro lado, los factores relacionados con la atención hospitalaria, como una falta de reconocimiento de alteraciones nutricionales, déficit en el entrenamiento nutricional del personal de salud, falla en el diagnóstico de desnutrición, inadecuado aporte calórico proteico y falta de reconocimiento sobre la importancia de la nutrición ${ }^{(5-7)}$.

En el mundo, la prevalencia de desnutrición hospitalaria se ha descrito entre $15 \%$ y $60 \%$, siendo en algunas regiones mayor a esta cifra ${ }^{(8-11)}$. En México, se tienen reportes de prevalencia de desnutrición que van de $26 \%$ a $55 \%$ de los pacientes ${ }^{(12,13)}$. Específicamente en la Ciudad de México, su prevalencia se ha encontrado entre $56 \%$ y $63 \%^{(14,15)}$.

Los métodos de tamizaje y diagnóstico nutricional permiten identificar a pacientes con desnutrición o riesgo nutricional hospitalizados. Una vez identificados, se procede a realizar una evaluación clínica - nutricional completa, así como el diseño de un plan de tratamiento nutricional. La utilidad del tamizaje y diagnóstico es limitada si no se acompaña de una intervención adecuada a la situación clínica del paciente.

La Valoración Global Subjetiva (VGS) es una herramienta diagnóstica descrita por Detsky en $1987^{(16)}$ y ampliamente utilizada en Latinoamérica ${ }^{(9)}$. Según una revisión de la literatura publicada en 2015, la VGS es reconocida como una herramienta válida para realizar el diagnóstico del estado nutricional de pacientes quirúrgicos y clínicos. La mayoría de estudios demuestran una capacidad similar o superior a los métodos tradicionales de antropometría o laboratorios bioquímicos. La VGS sigue utilizándose como herramienta de tamizaje nutricional considerándose en ocasiones, de manera errada, el gold estándar. Según la revisión, al comparar las herramientas de tamizaje nutricional con la VGS, estas son igual o mejores para detectar alteraciones del estado nutricional y su relación con peores resultados clínicos ${ }^{(17)}$. Todavía no hay estudios que comparen la validez de la VGS frente a los criterios diagnósticos de la Iniciativa de Liderazgo Global en Desnutrición (GLIM, por sus siglas en inglés) ${ }^{(18)}$.

Poco se sabe sobre la práctica actual de una intervención nutricional a los pacientes ya identificados con desnutrición o riesgo en los hospitales privados de la Ciudad de México. Los estudios publicados realizados en este país sobre prevalencia de desnutrición hospitalaria se han llevado a cabo en hospitales de instituciones públicas. A pesar de que se ha reportado la participación de instituciones privadas en grandes estudios como el ENHOLA (Estado Nutricional de los pacientes Hospitalizados en Latinoamérica) ${ }^{(8)}$, no existen publicaciones sobre desnutrición hospitalaria que únicamente involucren hospitales privados de México. El objetivo de este estudio es identificar el porcentaje de pacientes que recibieron tratamiento nutricional después del proceso de tamizaje al ingreso hospitalario en una institución privada de Ciudad de México.

\section{MÉTODO}

Estudio observacional, transversal y descriptivo. Se revisaron expedientes de pacientes internados en una 
institución privada de tercer nivel en la Ciudad de México, el Hospital Ángeles del Pedregal, dentro del área de hospitalización de terapia intermedia, medicina interna y cirugía, y sus especialidades, entre el 01 de enero y el 30 de junio de 2017.

Se realizó una muestra probabilística, aleatoria simple. Los criterios de inclusión fueron pacientes mayores de 18 años, con al menos 7 días de estancia intrahospitalaria y Valoración Global Subjetiva (VSG) dentro de las primeras 48 horas del ingreso hospitalario. Se excluyeron las pacientes embarazadas o en lactancia, con enfermedad terminal en manejo paliativo, pacientes en el área de terapia intensiva o urgencias, pacientes que a su ingreso estuviesen recibiendo apoyo nutricional especializado y aquellos cuyo diagnóstico de ingreso fuera algún tipo de desnutrición.

La VGS es la herramienta utilizada en la institución estudiada, evalúa el estado de nutrición de pacientes a través de la historia clínica y la exploración física. Con los resultados obtenidos, se clasifica en: $\mathrm{A}=$ bien nutrido, $\mathrm{B}$ = desnutrición moderada o sospecha de desnutrición y $\mathrm{C}$ $=$ gravemente desnutrido. Esta escala posee una sensibilidad de $96 \%$ - $97 \%$ y una especificidad de $82 \%$ - $83 \%{ }^{(19)}$. Dicha evaluación la realizan los nutriólogos del área de hospitalización. La intervención especializada (plan, tratamiento, seguimiento) la realizan tanto nutriólogos como médicos especialistas en nutrición clínica.

Durante el periodo de tiempo establecido se registraron 507 ingresos hospitalarios, ante lo cual se calculó una muestra representativa de 289 pacientes. La revisión de expedientes se realizó en revisión de expedientes en el área de archivo clínico: historia clínica, padecimiento actual, notas de evolución, indicaciones médicas.

Los datos se analizaron en el programa SPSS versión 24. Todas las variables fueron normales. Para las variables cualitativas los resultados se describen empleando frecuencias y porcentajes. Para las variables cuantitativas los resultados se describen empleando distribuciones porcentuales, medias, desviación estándar e IC $95 \%$.

El estudio fue aprobado por el Comité de Investigación y del Comité de Ética en Investigación del Hospital Ángeles del Pedregal, Ciudad de México, con número de registro 2481 .

\section{RESULTADOS}

Se analizaron 289 expedientes clínicos, de los cuales 91 no tenían VGS en las primeras 48 horas de ingreso hospitalario. De los 198 pacientes restantes, 32 pacientes tuvieron un resultado de bien nutrido, 63 pacientes desnutrición moderada o sospecha de desnutrición y 103 pacientes tuvieron desnutrición grave. Se encontró por lo tanto, una prevalencia de desnutrición de $83,8 \%$. Lo anterior se muestra en la Figura 1. En menores de 60 años, $73 \%$ presentó riesgo de desnutrición o desnutrición moderada o severa (VGS B o C), mientras que este mismo resultado se encontró en $93 \%$ de los pacientes mayores de 60 años.

Las características de los 198 pacientes incluidos fueron las siguientes: la media de edad fue 59,96 $\pm 17,53$

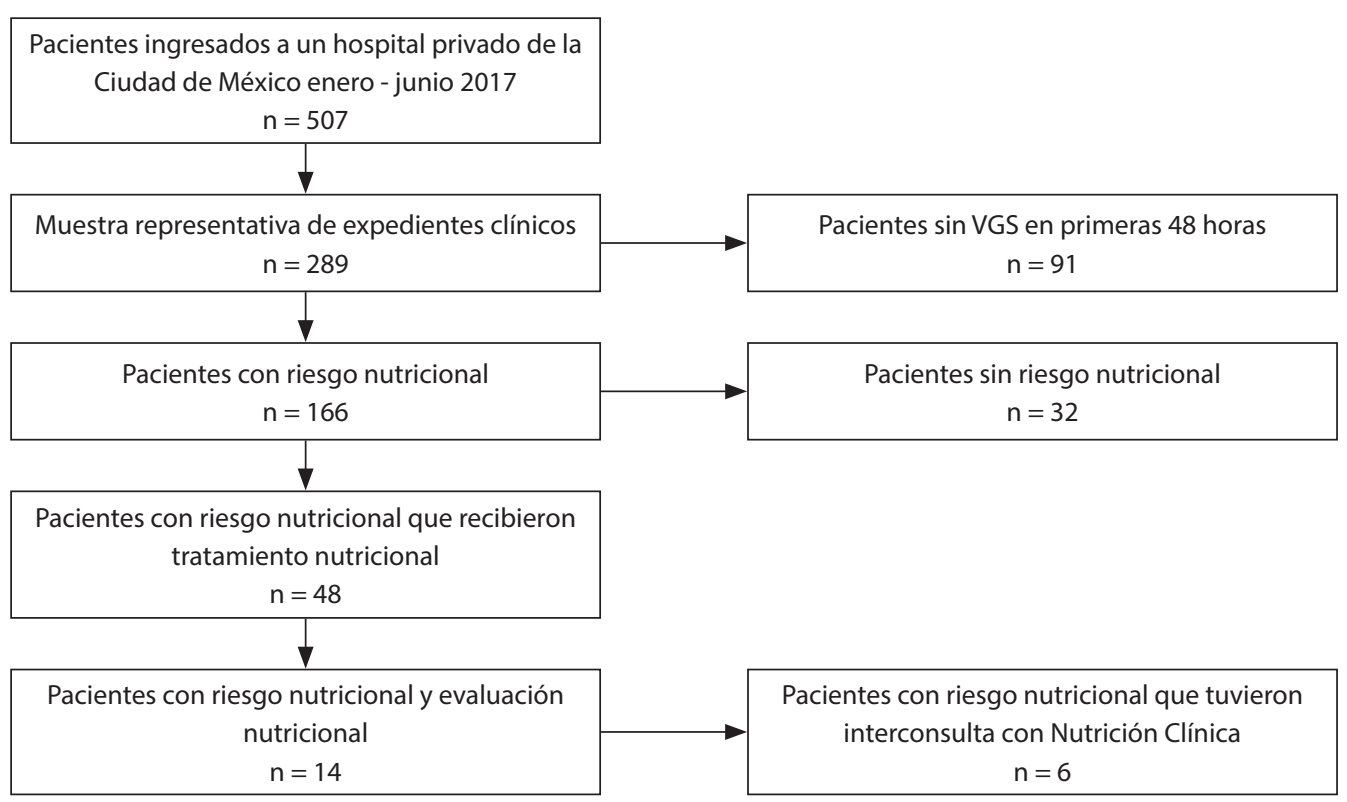

Figura 1. Diagrama de flujo del estudio. 
años. El 52,5 \% mujeres y el índice de masa corporal $25,53 \pm 4,89 \mathrm{Kg} / \mathrm{m}^{2}$. La media de los días de estancia hospitalaria fue 14,54 $\pm 12,09$ días.

De los pacientes con una VGS B o C (166 en total), a solo 14 se les hizo una evaluación nutricional integral. En el mismo grupo de pacientes con resultado de VGS $\mathrm{B}$ o $\mathrm{C}, 48$ pacientes recibieron algún tratamiento nutricional a los 7 días de estancia hospitalaria (Figura 2). El tratamiento administrado consistió en: terapia nutricional oral, 20 pacientes; nutrición enteral por sonda nasogástrica, 3; por sonda nasoyeyunal, 3; gastrostomía, 2; yeyunostomía, 1; y nutrición parenteral, 19.

En cuanto a los diagnósticos de ingreso hospitalario, se dividieron en grupos según su clasificación etiológica. Las patologías con mayor índice de desnutrición fueron las quirúrgicas, oncológicas, las relacionadas con el aparato respiratorio y de traumatología y ortopedia (Tabla 1). En ningún expediente clínico se encontró el diagnóstico de "Desnutrición".

Tabla 1. Riesgo nutricional por especialidades

\begin{tabular}{|l|c|c|c|}
\hline & VGS A & VGS B & VGS C \\
\hline $\begin{array}{l}\text { Cirugía } \\
(n=36)\end{array}$ & $3(8 \%)$ & $9(25 \%)$ & $24(67 \%)$ \\
\hline $\begin{array}{l}\text { Neumología } \\
(n=34)\end{array}$ & $(18 \%)$ & $(53 \%)$ & $(29 \%)$ \\
\hline $\begin{array}{l}\text { Oncología } \\
(n=24)\end{array}$ & $(0 \%)$ & $(33 \%)$ & $(67 \%)$ \\
\hline $\begin{array}{l}\text { Ortopedia/trauma } \\
(n=25)\end{array}$ & $5(20 \%)$ & $9(36 \%)$ & $11(44 \%)$ \\
\hline $\begin{array}{l}\text { Cardiología } \\
(n=21)\end{array}$ & $6(29 \%)$ & $4(19 \%)$ & $11(52 \%)$ \\
\hline $\begin{array}{l}\text { Neurología } \\
(n=18)\end{array}$ & $3(17 \%)$ & $5(28 \%)$ & $10(55 \%)$ \\
\hline $\begin{array}{l}\text { Otras (dermatología, } \\
\text { ginecología, urología) } \\
(n=18)\end{array}$ & $7(39 \%)$ & $2(11 \%)$ & $9(50 \%)$ \\
\hline $\begin{array}{l}\text { Nefrología } \\
(n=16)\end{array}$ & $1(6 \%)$ & $7(44 \%)$ & $8(50 \%)$ \\
\hline $\begin{array}{l}\text { Hematología } \\
(n=3)\end{array}$ & 1 & 1 \\
\hline
\end{tabular}

VGS: Valoración global subjetiva

\section{DISCUSIÓN}

El propósito de este estudio es identificar la prevalencia de pacientes con desnutrición o riesgo de desnutrición y conocer qué porcentaje de ellos recibe tratamiento nutricional en un hospital privado de la Ciudad de México. El principal resultado fue que 83,8 \% de la población presentó a su ingreso hospitalario algún grado de desnutrición según la herramienta VGS. Esta cifra es mayor que el promedio reportado en las publicaciones de México, que va de $25 \%$ a $63 \%$. Lo anterior demuestra que la prevalencia de la desnutrición hospitalaria es alta y existe en todos los ámbitos de servicios de salud.

En nuestro estudio la mayoría de los pacientes (71\%) no recibió ningún tratamiento para su patología nutricional. Además, $32,5 \%$ de la muestra inicial no contó con una VGS a su ingreso, o se realizó en un tiempo mayor de 48 horas.

De los pacientes con riesgo de desnutrición o con desnutrición moderada o severa, solo a $8,4 \%$ se le realizó una evaluación nutricional completa, es decir, una valoración clínica y bioquímica que permitiera hacer un diagnóstico del estado nutricional y determinar un plan terapéutico. Es importante resaltar que la mayoría $(91,6 \%)$ de los pacientes no tuvo esta evaluación ni los beneficios que representa.

Del total de pacientes con riesgo de desnutrición o desnutrición ya sea moderada o severa, a solo $28,9 \%$ se le ofreció algún tratamiento nutricional (suplemento, nutrición enteral o nutrición parenteral) en el día 7 de hospitalización. Estos resultados se pueden contrastar con los encontrados en la literatura, siendo similares en cuanto a la baja implementación del tratamiento nutricional. El análisis de resultados del estudio nutritionDay entre 2007 y 2008 en 21.007 de 25 países encontró que solo $20 \%$ de pacientes diagnosticados con riesgo nutricional, recibieron algún suplemento proteico ${ }^{(20)}$. Un estudio retrospectivo en 8.541 pacientes hospitalizados en medicina interna en un hospital suizo comparó la práctica del cuidado nutricional entre los años 2103 y 2014 encontrando que menos de la mitad de los pacientes con riesgo nutricional recibieron algún tipo de tratamiento nutricional siendo de $46,9 \%$ y de $40,3 \%$ respectivamente ${ }^{(21)}$. Kondrup et al., en 750 pacientes de tres hospitales de Dinamarca, reportaron que únicamente $25 \%$ de los pacientes con riesgo nutricional recibieron una adecuada cantidad de energía y proteína. Además, solo $30 \%$ de estos pacientes contó con un monitoreo del peso y de la ingesta de alimentos ${ }^{(22)}$. En un estudio transversal multicéntrico holandés se encontró que menos de $50 \%$ de todos los pacientes desnutridos hospitalizados, en residencias geriátricas y en atención domiciliaria recibieron terapia nutricional ${ }^{(23)}$.

Se recomienda que todos los pacientes se beneficien de un cuidado nutricional óptimo y oportuno el cual 
debe iniciarse con el tamizaje nutricional en las primeras 48 horas del ingreso hospitalario como se reconoce en el Principio 2 de la Declaración de Cartagena ${ }^{(24)}$.

El presente estudio retrospectivo y descriptivo no permite determinar causalidad. Sin embargo, consideramos que la baja implementación del tamizaje, la evaluación nutricional, seguimiento y tratamiento nutricional se podría explicar por la falta de una cultura institucional que reconozca la importancia del cuidado nutricional y de un manejo interdisciplinario. Esto se puede observar en la falta de interconsultas al servicio de Nutrición Clínica, formado tanto por nutriólogos como por médicos con especialización en Nutrición Clínica. De los expedientes revisados de pacientes con riesgo nutricional o desnutrición, solo se encontraron 6 interconsultas al servicio de Nutrición Clínica por parte del servicio tratante. Es decir, únicamente se buscó apoyo de personal especializado en 3,6 \% de los pacientes con desnutrición (Figura 2). Estos datos se contraponen con los publicados por Rosas González et al., donde encontraron que en el Hospital General de México (institución pública) a $81,42 \%$ de los pacientes hospitalizados con riesgo nutricional le solicitaron interconsulta al servicio de Nutrición Clínica por parte del servicio tratante ${ }^{(25)}$.

Es probable que por ser un hospital privado, exista cierta limitación en el empleo de nutrición especializada por los gastos que esta genera. Sin embargo, teniendo en cuenta la literatura, el beneficio supera los costos, ya que está descrito en varias publicaciones el ahorro económico que representa el abordaje multidisciplinario del soporte nutricional al prevenir complicaciones costosas y mayor número de días de estancia hospitalaria $^{(26-29)}$.
En el expediente clínico de todos los pacientes, se observó ausencia de diagnóstico de desnutrición al egreso; problema que se identifica en otros estudios ya que la literatura reporta que solo entre $1 \%$ y $13 \%$ de los pacientes con diagnóstico de desnutrición tienen registrado en su expediente clínico este diagnóstico $^{(30,31)}$. Se debe reconocer y dar la importancia que este merece para poder tratarlo.

La relevancia de este estudio recae en que son pocos los estudios publicados sobre desnutrición hospitalaria en hospitales privados de México, lo que abre un panorama de áreas de oportunidad para la mejor atención de la salud en la población mexicana. Con lo anterior se aportan datos que ayudan a fomentar la importancia del tratamiento nutricional adecuado en el paciente hospitalizado en el ámbito privado.

Dentro de las limitantes se encuentra el número reducido de expedientes revisados en el periodo de tiempo establecido. Esto pudiera explicar la alta prevalencia de desnutrición encontrada.

\section{CONCLUSIÓN}

Los resultados de este estudio muestran una alta prevalencia de desnutrición en los pacientes que ingresaron a este hospital privado en la Ciudad de México. La mayoría de los pacientes con riesgo de desnutrición y con desnutrición moderada o severa no reciben evaluación, seguimiento ni tratamiento nutricional especializado. Estos hallazgos significan un área de oportunidad para intervenir en la mejor atención del paciente hospitalizado, creando nuevas y mejores estrategias para el tratamiento del paciente con alteraciones nutricionales.

Con los datos obtenidos podemos sugerir campañas de educación sobre nutrición y su importancia en el

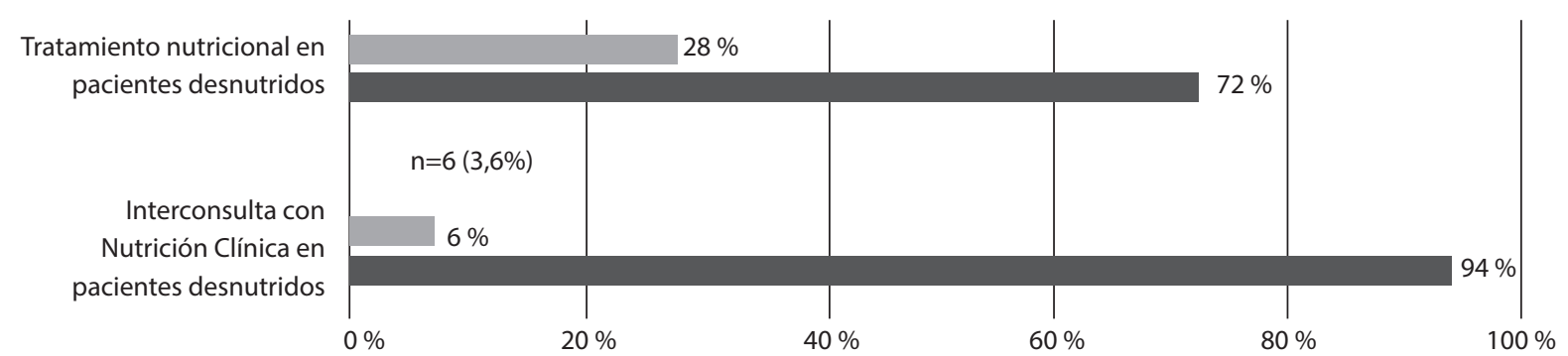

Figura 2. Tratamiento nutricional e interconsulta con Nutrición Clínica en pacientes con desnutrición. Las barras en gris claro muestran el \% de pacientes que tuvieron interconsulta con el equipo de nutrición; las barras en gris oscuro muestran el \% de pacientes que no tuvieron interconsulta con el equipo de nutrición. 
ámbito hospitalario a todo el personal de la salud involucrado en la atención de pacientes. Además, enfatizar en la existencia de un equipo de Nutrición integrado por enfermeras, farmacéuticos, nutriólogos y médicos especialistas en Nutriología Clínica para lograr una completa atención multidisciplinaria del paciente.

\section{Financiación}

El presente estudio no tuvo financiación.

\section{Conflicto de intereses}

Los autores declaran no tener ningún conflicto de intereses.

\section{Agradecimientos}

Agradecemos al Comité de Investigación y al Comité de Ética en Investigación de Operadora de Hospitales Ángeles S.A. de C.V.

\section{Declaración de autoría}

Redacción del artículo: Adriana Vázquez Callejas; Realización y desarrollo de la investigación: Adriana Vázquez Callejas, Quetzalina Flores Fajardo, Jorge Chirino Romo; Obtención de datos: Adriana Vázquez Callejas, Quetzalina Flores Fajardo; Análisis de los resultados: Adriana Vázquez Callejas, Jorge Chirino Romo; Interpretación de los resultados: Adriana Vázquez Callejas, Jorge Chirino Romo. Todos los autores revisaron el artículo y validaron su versión final.

\section{Referencias bibliográficas}

1. Cederholm T, Barazzoni R, Austin P, Ballmer P, Biolo G, Bischoff S, et al. ESPEN guidelines on definitions and terminology of clinical nutrition. Clin Nutr. 2017;36(1):49-64.

2. Norman K, Pichard C, Lochs H, Pirlich M. Prognostic impact of disease-related malnutrition. Clin Nutr. 2008;27(1):5-15.

3. Barker L, Gout B, Crowe T. Hospital Malnutrition: Prevalence, Identification and Impact on Patients and the Healthcare System. Int J Environ Res Public Health. 2011;8(2):514-27.

4. Amaral T, Matos L, Teixeira M, Tavares M, Alvares L, Antunes A. Undernutrition and associated factors among hospitalized patients. Clin Nutr. 2010;29(5):580-5.

5. Fuchs V, MostkoffD, Guitérrez G, Amancio O. Estado nutricio en pacientes internados en un hospital público de la Ciudad de México. Nutr Hosp. 2008; 23(3):294-303.

6. Kubrack C, Jensen L. Malnutrition in acute care patients: a narrative review. Int J Nurs Stud. 2007; 44(6):1036-54.
7. Keller H, Allard J, Vesnaver E, Laporte M, Gramlich L, Bernier $\mathrm{P}$, et al. Barriers to food intake in acute care hospitals: a report of the Canadian Malnutrition Task Force. J Hum Nutr Diet. 2015;28(6):546-57.

8. Castillo Pineda JC, Gómez García A, Velasco N, Díaz Pizarro Graf JI, Matos Adamés A, Miján de la Torre A. Nutritional assessment of hospitalized patients in Latin America: Association with prognostic variables. The ENHOLA study. Nutr Hosp. 2016;33(3):655-62.

9. Correia MI, Perman M, Waitzberg D. Hospital malnutrition in Latin America: A systematic review. Clin Nutr. 2017;36(4):958-67.

10. Wysynski DF, Perman M, Crivelli A. Prevalence of hospital malnutrition in Argentina: Preliminary results of a population-based study. Nutrition. 2003; 19(2):115-9.

11. Waitzberg DL, Caiaffa WT, Correia MI. Hospital malnutrition: the Brazilian national survey (IBRANUTRI): a study of 4000 patients. Nutrition. 2001; 17(7-8):573-80.

12. Pérez-Flores JE, Chávez-Tostado $M$, Larios-del-Toro YE, García-Rentería J, Rendón-Félix J, Salazar-Parra M, et al. Evaluación del estado nutricional al ingreso hospitalario y su asociación con la morbilidad y mortalidad en pacientes mexicanos. Nutr Hosp. 2016;33(4):872-8.

13. Jaimes E, Zúñiga MG, Medina A, Reyes D, Cruz A. Prevalencia de riesgo nutricional en un hospital de segundo nivel en el Estado de México. Nutr Clín Diet Hosp. 2016;36(4):111-6.

14. Pérez Cruz E, Villalobos Ruiz SB. Desnutrición hospitalaria: Prevalencia en el Hospital Juárez de México. Rev Hosp Jua Mex. 2010;77(4):234-8.

15. Pérez MT, Serralde A, Reyes-Ramírez AL, Alfonso-Baruch E, Gulias-Herrero A, Castillo-Martínez L. Prevalence of Malnutrition at admission in hospitalized adults at INCMNSZ in Mexico City. Rev Mex Endocrinol Metab Nutr. 2017;4:12-6.

16. Detsky AS, McLaughlin JR, Baker JP, Johnston N, Whittaker $\mathrm{S}$, Mendelson RA, et al. What is subjective global assessment of nutritional status? JPEN J Parenter Enteral Nutr. 1987;11(1):8-13.

17. Da Silva Fink J, Daniel de Mello P, Daniel de Mello E. Subjective global assessment of nutritional status - A systematic review of the literature. Clin Nutr. 2015;34(5):785-92.

18. Cederholm T, Jensen GL, Correia MITD, Gonzalez MC, Fukushima R, Higashiguchi T, et al. GLIM criteria for the diagnosis of malnutrition- A consensus report from the global clinical nutrition community. Clin Nutr. 2019;38(1):1-9.

19. Ravasco P, Anderson H, Mardones F. Métodos de valoración del estado nutricional. Nutr Hosp. 2010;25(3):57-66.

20. Schindler K, Pernicka E, Laviano A, Howard P, Schütz T, Bauer $\mathrm{P}$, et al. How nutritional risk is assessed and managed in European hospitals: a survey of 21,007 patient findings from the 2007-2008 cross-sectional nutritionDay survey. Clin Nutr. 2010;29(5):552-9.

21. Khalatbari-Soltani S, Marques-Vidal P. Impact of nutritional risk screening in hospitalized patients on management, 
outcome and costs: A retrospective study. Clinical Nutrition. 2016;35(6):1340-6.

22. Kondrup J, Johansen N, Plum LM, Bak L, Larsen IH, Martinsen A, et al. Incidence of nutritional risk and causes of inadequate nutritional care in hospitals. Clin Nutr Edinb Scotl. 2002;21:461-8.

23. Meijers JM, Halfens RJ, van Bokhorst-de van der Schueren MA, Dassen T, Schols JM. Malnutrition in Dutch health care: prevalence, prevention, treatment, and quality indicators. Nutrition. 2009;25:512-9.

24. Cardenas D, Bermúdez $\mathrm{CH}$, Echeverri S, Perez A, Puentes M, Lopez $\mathrm{M}$, et al. DECLARACIÓN DE CARTAGENA. Declaración Internacional sobre el Derecho al Cuidado Nutricional y la Lucha contra la Malnutrición. Nutr Hosp. 2019;36(4):974-98. http://dx.doi.org/10.20960/nh.02701.

25. Rosas González E, Álvarez K, Bejarano M, Fuchs V. Relación entre el riesgo nutricional y el número de interconsultas realizadas al servicio de nutrición clínica según el servicio hospitalario. Rev. Nutr. Clin. Metab. 2018;1(2): 44-8.

26. Banks MD, Graves N, Bauer JD, Ash S. Cost effectiveness of nutrition support in the prevention of pressure ulcer in hospitals. Eur J Clin Nutr. 2013; 67(1): 42-6.
27. Hudson L, Chittams J, Griffith C, Compher C. Malnutrition Identified by Academy of Nutrition and Dietetics/American Society for Parenteral and Enteral Nutrition Is Associated With More 30-Day Readmissions, Greater Hospital Mortality, and Longer Hospital Stays: A Retrospective Analysis of Nutrition Assessment Data in a Major Medical Center. JPEN J Parenter Enteral Nutr. 2018; 42(5):892-7.

28. Correia MI, Waitzberg DL. The impact of malnutrition on morbidity, mortality, length of hospital stay and costs evaluated through a multivariate model analysis. Clin Nutr. 2003; 22(3): 235-9.

29. Doig GS, Simpson F. Sweetman EA, Finer SR, Cooper DJ, Heighes PT, et al. Early parenteral nutrition in critically ill patients with short-term relative contraindications to early enteral nutrition: a randomized controlled trial. JAMA. 2013; 309(20): 2130-8.

30. Singh H, Watt K, Veitch R, Cantor M, Duerksen DR. Malnutrition is prevalent in hospitalized medical patients: Are housestaff identifying the malnourished patient? Nutrition. 2006;22(4):350-4.

31. McWhirter JP, Pennington CR. Incidence and recognition of malnutrition in hospital. Clin Nutr. 1994;308(6934):945- 8. 